\title{
Connections between nonrotating, slowly rotating, and rapidly rotating turbulent convection transport scalings
}

\author{
Jonathan M. Aurnou $\odot,{ }^{1, *}$ Susanne Horn $\odot,{ }^{2}$ and Keith Julien $\odot^{3}$ \\ ${ }^{1}$ University of California at Los Angeles, Los Angeles, California 90095-1567, USA \\ ${ }^{2}$ Coventry University, Coventry CV1 5FB, United Kingdom \\ ${ }^{3}$ University of Colorado at Boulder, Boulder, Colorado 80309, USA
}

(Received 22 February 2020; accepted 7 September 2020; published 21 October 2020; corrected 30 March 2021)

In this paper, we investigate and develop scaling laws as a function of external nondimensional control parameters for heat and momentum transport for nonrotating, slowly rotating, and rapidly rotating turbulent convection systems, with the end goal of forging connections and bridging the various gaps between these regimes. Two perspectives are considered, one where turbulent convection is viewed from the standpoint of an applied temperature drop across the domain and the other with a viewpoint in terms of an applied heat flux. While a straightforward transformation exists between the two perspectives, indicating equivalence, it is found the former provides a clear set of connections that bridge between the three regimes. Our generic convection scalings, based upon an inertial-Archimedean balance, produce the classic diffusion-free scalings for the nonrotating limit and the slowly rotating limit. This is characterized by a free-falling fluid parcel on the global scale possessing a thermal anomaly on par with the temperature drop across the domain. In the rapidly rotating limit, the generic convection scalings are based on a Coriolis-inertial-Archimedean (CIA) balance, along with a local fluctuating-mean advective temperature balance. This produces a scenario in which anisotropic fluid parcels attain a thermal wind velocity and where the thermal anomalies are greatly attenuated compared to the total temperature drop. We find that turbulent scalings may be deduced simply by consideration of the generic nondimensional transport parameters-local Reynolds $\operatorname{Re}_{\ell}=U \ell / \nu$; local Péclet $\mathrm{Pe}_{\ell}=U \ell / \kappa$; and Nusselt number $\mathrm{Nu}=U \vartheta /(\kappa \Delta T / H)$-through the selection of physically relevant estimates for length $\ell$, velocity $U$, and temperature scales $\vartheta$ in each regime. Emergent from the scaling analyses is a unified continuum based on a single external control parameter, the convective Rossby number, $\operatorname{Ro}_{C}=\sqrt{g \alpha \Delta T / 4 \Omega^{2} H}$, that strikingly appears in each regime by consideration of the local, convection-scale Rossby number $\operatorname{Ro}_{\ell}=U /(2 \Omega \ell)$. Thus we show that $\mathrm{Ro}_{C}$ scales with the local Rossby number $\mathrm{Ro}_{\ell}$ in both the slowly rotating and the rapidly rotating regimes, explaining the ubiquity of $\mathrm{Ro}_{C}$ in rotating convection studies. We show in non-, slowly, and rapidly rotating systems that the convective heat transport, parametrized via $\mathrm{Pe}_{\ell}$, scales with the total heat transport parameterized via the Nusselt number $\mathrm{Nu}$. Within the rapidly rotating limit, momentum transport arguments generate a scaling for the system-scale Rossby number, $\mathrm{Ro}_{H}$, that, recast in terms of the total heat flux through the system, is shown to be synonymous with the classical flux-based CIA scaling, Ro ${ }_{\text {CIA. These, in turn, are }}$ then shown to asymptote to $\mathrm{Ro}_{H} \sim \mathrm{Ro}_{\text {CIA }} \sim \mathrm{Ro}_{C}^{2}$, demonstrating that these momentum transport scalings are identical in the limit of rapidly rotating turbulent heat transfer.

DOI: 10.1103/PhysRevResearch.2.043115

\section{INTRODUCTION}

Accurate parametrizations are ubiquituously sought for the turbulent transport properties of fluid dynamical systems. In buoyancy-driven convection systems, the heat and momentum transport properties are the main foci of such investigations [1-4]. These transport estimates are essential for understanding the possible behaviors of a given system and for extrapolating these behaviors to extreme industrial,

\footnotetext{
*aurnou@g.ucla.edu
}

Published by the American Physical Society under the terms of the Creative Commons Attribution 4.0 International license. Further distribution of this work must maintain attribution to the author(s) and the published article's title, journal citation, and DOI. geophysical and astrophysical settings that are difficult to simulate directly (e.g., Refs. [5-13]).

In the Rayleigh-Bénard convection systems considered here, warmer fluid is maintained at the base of the fluid layer and colder fluid is maintained at the top of the layer, defined with respect to the gravity vector $g$ that is parallel to the background temperature gradient. In addition, our system is rotating at angular velocity $\boldsymbol{\Omega}$ that is oriented in the axial $\hat{\boldsymbol{e}}_{z}$ direction. This system is gravitationally unstable and drives buoyant convective flows across the fluid layer that advect both heat and momentum. We describe this system generally throughout this paper, but it can be thought of as an extended plane layer [14], a finite cylinder [15], or a spherical shell of fluid [16].

A scaling analysis is presented using generic scales for the characteristic fluid properties occurring in the nonrotating, slowly rotating, and rapidly rotating turbulent limits. This 
analysis generates a large-scale, free-fall flow regime in the nonrotating (NRL) and slowly rotating limits (SRL), and a small-scale, thermal wind flow in the rapidly rotating limit (RRL). The generic nature of our scaling analysis allows us to provide connections between the different regimes. For instance, we show that the convective Rossby number, $\operatorname{Ro}_{C}$, arises ubiquitously in scaling estimates for turbulent rotating convection, both in the rapidly rotating and slowly rotating end-member limits. Further, $\operatorname{Ro}_{C}$ is shown to be equivalent to $\mathrm{Ro}_{\ell}$, which describes the Rossby number for the rotating flow dynamics on the local convective scale. The rotating scalings developed show how numerous heat and momemtum transport laws can all be inter-related via integer powers of $\mathrm{Ro}_{C}$ (or, synonymously, $\mathrm{Ro}_{\ell}$ ), thus providing novel ties between the different scaling regimes.

\section{GOVERNING EQUATIONS AND PARAMETERS}

The governing equations of rotating thermal convection in an Oberbeck-Boussinesq fluid are

$$
\begin{aligned}
\partial_{t} \boldsymbol{u}+\boldsymbol{u} \cdot \nabla \boldsymbol{u}+2 \boldsymbol{\Omega} \times \boldsymbol{u} & =-\nabla p+\boldsymbol{g} \alpha \theta+v \nabla^{2} \boldsymbol{u}, \\
\partial_{t} \theta+(\boldsymbol{u} \cdot \nabla \theta)^{\prime} & =\left(\hat{\boldsymbol{e}}_{\boldsymbol{g}} \cdot \boldsymbol{u}\right) \partial_{g} \bar{T}+\kappa \nabla^{2} \theta, \\
\partial_{t} \bar{T}+\nabla \cdot \overline{(\boldsymbol{u} \theta)} & =\kappa \nabla^{2} \bar{T} \\
\nabla \cdot \boldsymbol{u} & =0
\end{aligned}
$$

e.g., Refs. [17]. Other effects are not considered here, such as those due to magnetic fields [18], centrifugal buoyancy [19], and non-Oberbeck-Boussinesqness [20]. In the Navier-Stokes Eq. (1a), the velocity vector is $\boldsymbol{u}$, the angular rotation velocity along the axial coordinate $z$ is $\boldsymbol{\Omega}, p$ is the modified pressure, $\alpha$ is the thermal expansivity, $\boldsymbol{g}$ is the gravity vector, and $v$ is the fluid's kinematic viscosity.

Temperature is $T=\bar{T}+\theta$, where the overbar denotes averaging over surfaces perpendicular to $g$. Thus, $\bar{T}$ is the laterally averaged temperature and $\theta$ is the temperature fluctuation. Equation (1b) is the fluctuating temperature evolution equation and Eq. (1c) describes the evolution of the laterally averaged temperature field. In the fluctuating temperature Eq. (1b), we use the abbreviated notation $(\boldsymbol{u} \cdot \nabla \theta)^{\prime}=$ $\boldsymbol{u} \cdot \boldsymbol{\nabla} \theta-\nabla \cdot \overline{(\boldsymbol{u} \theta)}$. Convective motions in this system are driven by an unstable, system-scale temperature gradient $\partial_{g} \bar{T}=\mathcal{O}(\Delta T / H)$ measured in the direction of gravity $\hat{\boldsymbol{e}}_{\boldsymbol{g}}$, where $\Delta T$ is the temperature drop across the fluid layer of system depth $H$. Here $\Delta T$ is sustained either via fixed temperature boundaries or via an applied heat flux $Q$ [21]. Depending on the setup, $\hat{\boldsymbol{e}}_{\boldsymbol{g}}$ can be oriented in the axial direction $\hat{\boldsymbol{e}}_{z}$ [22], the cylindrically radial direction $\hat{\boldsymbol{e}}_{\boldsymbol{s}}$ [23], or the spherically radial direction $\hat{\boldsymbol{e}}_{\boldsymbol{r}}$ [16].

Here we take the characteristic convective velocity to be $U$, the characteristic length scale to be $\ell$, and the characteristic temperature anomaly to be $\vartheta$. The SRL is defined such that the inertial forces greatly exceed the Coriolis force:

$$
\boldsymbol{u} \cdot \nabla \boldsymbol{u} \gg 2 \boldsymbol{\Omega} \times \boldsymbol{u} \longrightarrow \frac{U^{2}}{\ell} \gg 2 \Omega U .
$$

The ratio of these terms, the so-called local Rossby number defined with the characteristic scales of the convection, is

$$
\operatorname{Ro}_{\ell} \equiv \frac{U}{2 \Omega \ell} \gg 1 \text {. }
$$

In the RRL of Rayleigh-Bénard convection, the Coriolis forces dominate over the inertial forces,

$$
\boldsymbol{u} \cdot \nabla \boldsymbol{u} \ll 2 \boldsymbol{\Omega} \times \boldsymbol{u} \quad \longrightarrow \quad \frac{U^{2}}{\ell} \ll 2 \Omega U .
$$

Thus,

$$
\operatorname{Ro}_{\ell} \equiv \frac{U}{2 \Omega \ell} \ll 1
$$

We note then that the local Rossby number estimates the strength of inertial advection using the estimated convective velocity and length scales considered, normalized by the Coriolis acceleration.

We are interested in ascertaining turbulent scaling laws for the heat transported across the system scale $H$ and for the local momentum and heat transport carried by the fluid motions occurring on the convective scale $\ell$. The system-scale heat transport is measured by the Nusselt number,

$$
\mathrm{Nu}=\frac{Q H}{\rho c_{P} \kappa \Delta T} \sim \frac{U \vartheta H}{\kappa \Delta T},
$$

where $\rho$ is the fluid's density and $c_{P}$ its specific heat capacity. Here $Q \sim \rho c_{P} U \vartheta$ is the total (superadiabatic) heat flux per unit area, which we assume is dominated by the turbulent convective transport component (i.e., $\mathrm{Nu} \gg 1$ ). The momentum and heat transported on the characteristic convective scale is estimated via the local Reynolds and Péclet numbers

$$
\operatorname{Re}_{\ell}=\frac{U \ell}{v}, \quad \operatorname{Pe}_{\ell}=\frac{U \ell}{\kappa} .
$$

The $\mathrm{Nu}, \mathrm{Re}_{\ell}$, and $\mathrm{Pe}_{\ell}$ transport scalings will be formulated in terms of Eqs. (1)'s nondimensional control parameters, which are the Prandtl, Rayleigh, and Ekman numbers [4]. The Prandtl number describes the fluid's thermophysical properties,

$$
\operatorname{Pr}=\frac{v}{\kappa},
$$

where $\kappa$ and $v$ are the thermal diffusivity and kinematic viscosity, respectively. The Ekman number, Ek, gives the estimated ratio of system-scale viscous and Coriolis forces:

$$
\mathrm{Ek}=\frac{v}{2 \Omega H^{2}} .
$$

The Rayleigh number estimates the strength of the buoyancy forcing:

$$
\mathrm{Ra}=\frac{g \alpha \Delta T H^{3}}{\nu \kappa} .
$$

The nondimensional buoyancy forcing will also be presented in three alternative forms. The first of these is in terms of the flux Rayleigh number, based on the heat flux through the system:

$$
\mathrm{Ra}_{F}=\mathrm{RaNu}=\frac{g \alpha Q H^{4}}{\rho c_{P} \nu \kappa^{2}} .
$$

Following Christensen [24] and Christensen and Aubert [25], the second form is given in terms of the rotating, diffusivityfree, so-called modified flux Rayleigh number,

$$
\mathrm{Ra}_{F}^{*}=\frac{\mathrm{Ra}_{F} \mathrm{Ek}^{3}}{\operatorname{Pr}^{2}}=\frac{g \alpha Q}{8 \rho c_{P} \Omega^{3} H^{2}} .
$$



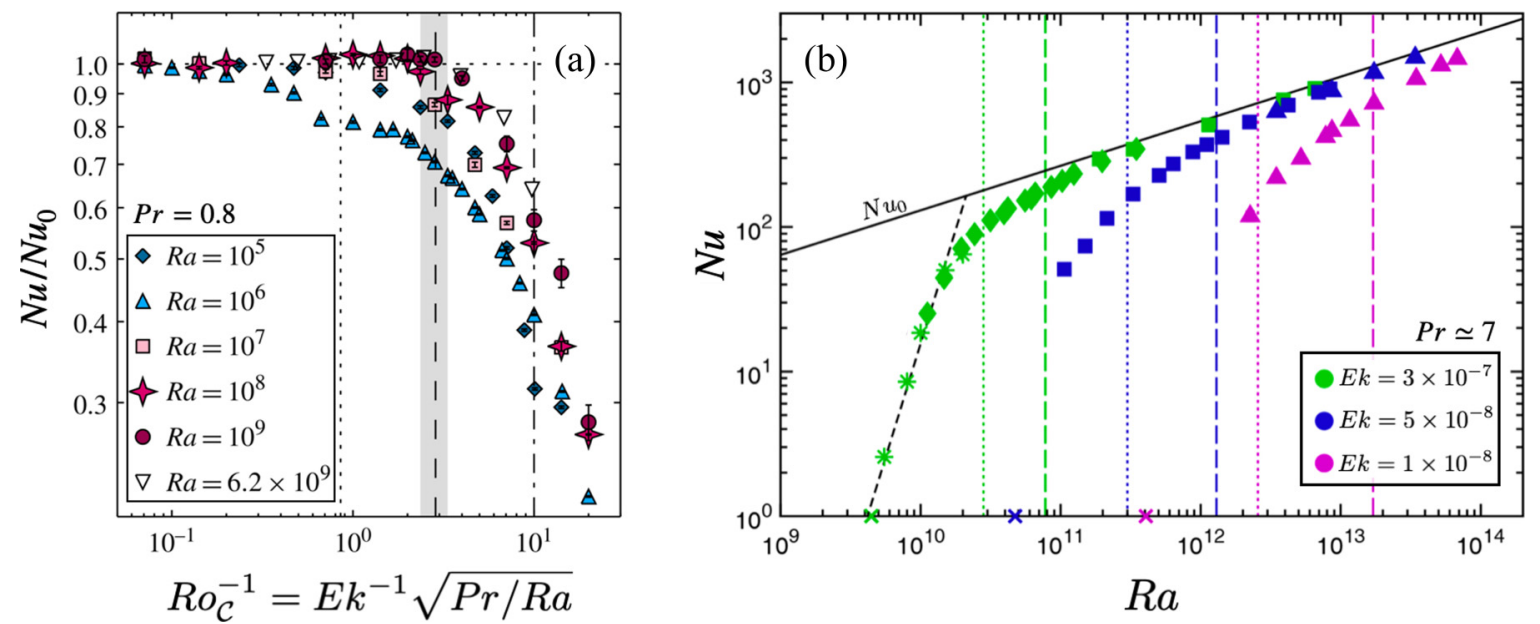

FIG. 1. Rotating convection heat transport surveys carried out in the slow rotating and rapidly rotating frameworks. (a) Slowly rotating approach: Fixed Ra data shingles from the numerical study of Horn and Shishkina [50]. For each data shingle, the vertical temperature difference $\Delta T(\propto \mathrm{Ra})$ is fixed and the angular rotation rate $\Omega\left(\propto \mathrm{Ek}^{-1}\right)$ is varied. (b) Rapidly rotating approach: Fixed Ek data shingles from the laboratory-numerical study of Cheng et al. [52]. Here $\Omega$ is fixed and $\Delta T$ is varied along each data shingle. The colored x symbols mark each Ek-shingle's $\mathrm{Ra}_{\text {crit }}$ value. The corresponding nonrotating heat transfer efficiency is denoted by $\mathrm{Nu}_{0}$.

(The oceanic and atmospheric communities write these flux-based parameters in terms of the buoyancy flux $\mathcal{B}=$ $g \alpha Q /\left(\rho c_{P}\right)$ and the Coriolis parameter $f=2 \Omega$ [26]. Then $\mathrm{Ra}_{F}=\mathcal{B} H^{4} / \nu \kappa^{2}$ and $\mathrm{Ra}_{F}^{*}=\mathcal{B} / f^{3} H^{2}$.) The third form is the convective supercriticality,

$$
\widetilde{\mathrm{Ra}}=\mathrm{Ra} / \mathrm{Ra}_{\text {crit }},
$$

where $\mathrm{Ra}_{\text {crit }}$ is the critical Rayleigh number above which buoyancy-driven fluid motions first onset in a given convection system [27-29]. Thermal convection is active whenever $\widetilde{\mathrm{Ra}} \geqslant 1$. No convection occurs for $\widetilde{\mathrm{Ra}}<1$, unless a subcritical branch also exists, giving rise to a hysteretic bistable state. This has been found in low Pr, rapidly rotating convection studies in spheres, such as in Guervilly and Cardin [30] and Kaplan et al. [31]. The critical Rayleigh number is approximately $10^{3}$ in nonrotating systems [32]. More specifically, $\mathrm{Ra}_{\text {crit }}=1708$ for no-slip mechanical boundary conditions in a nonrotating, horizontally infinite layer of fluid. In contrast, in a rotating plane layer of $\operatorname{Pr} \gtrsim 0.67$ fluid, the critical Rayleigh number is a strong function of the rotation rate and fluid viscosity,

$$
\mathrm{Ra}_{\text {crit }} \simeq 8.7 \mathrm{Ek}^{-4 / 3}
$$

and convection onsets in the form of stationary modes. In lower Prandtl number fluids such that $\operatorname{Pr} \lesssim 0.67$, convection first develops via oscillatory modes $[27,33,34]$ and the critical Rayleigh number in a plane layer is $\mathrm{Ra}_{\text {crit }} \simeq 17.4(\mathrm{Ek} / \mathrm{Pr})^{-4 / 3}$ $[35,36]$. Thus, in plane-layer geometries, $\mathrm{Ra}_{\text {crit }}$ depends on the rotation rate and the fluid's thermal diffusivity in low Pr fluids. In rotating spherical geometries, the onset is always to Prdependent oscillatory convection [37]. Although Pr can affect $\mathrm{Ra}_{\text {crit }}$ in rotating fluids [38], it does not affect the outcome of our analyses, since all the diffusion coefficients drop out of the final expressions. For simplicity, then, we will choose to consider only the moderate Pr relationship $\mathrm{Ra}_{\text {crit }} \sim \mathrm{Ek}^{-4 / 3}$ from here onward.
Lastly, we present the convective Rossby number, $\operatorname{Ro}_{C}$, which arises ubiquitously in studies of rotating convection. This nondimensional parameter estimates the ratio of buoyancy and Coriolis forces and is commonly defined as

$$
\operatorname{Ro}_{C} \equiv \sqrt{\frac{g \alpha \Delta T}{4 \Omega^{2} H}}=\sqrt{\frac{\mathrm{RaEk}^{2}}{\operatorname{Pr}}} .
$$

The convective Rossby number is taken to be the essential control parameter in many studies of rotating convection [13,39-44], and is also claimed to control numerous transitions in rotating convection behavior [45-51]. Further, in many rotating convection and dynamo studies, the buoyancy forcing is parameterized in terms of the square of the convective Rossby number, although it is referred to there as the modified Rayleigh number, $\mathrm{Ra}^{*}=\mathrm{Ro}_{C}^{2}$ [24].

\section{Parameter surveys}

Within the fluid physics community, rotating convection studies often take the NRL as their philosophical starting point. This assumes an inertial velocity scale and then the inertial turbulence is perturbed with increasing rotational effects. Within this buoyancy-dominated framework, surveys are carried out at various fixed values of the buoyancy forcing, e.g., fixed $\mathrm{Ra} \propto \Delta T$, while the angular rotation rate of the system $\Omega$ is systematically increased [42,44,53]. An example of this approach is shown in Fig. 1(a), which is adapted from the numerical investigation of Horn and Shishkina [50]. Six different cuts through parameter space are shown, with each data "shingle" made at a fixed $\mathrm{Ra}$ value as shown in the legend box [54]. The control parameter displayed along the abscissa is $1 / \operatorname{Ro}_{C}$, which in this case varies only as a function of the nondimensional rotation rate of the system $\mathrm{Ek}^{-1} \propto \Omega$. The ordinate shows the Nusselt number, $\mathrm{Nu}$, normalized by its NRL value at each $\mathrm{Ra}$ value, $\mathrm{Nu}_{0}(\mathrm{Ra})$.

In the geophysical and astrophysical fluid dynamics communities, it is typically argued that convection occurs within 


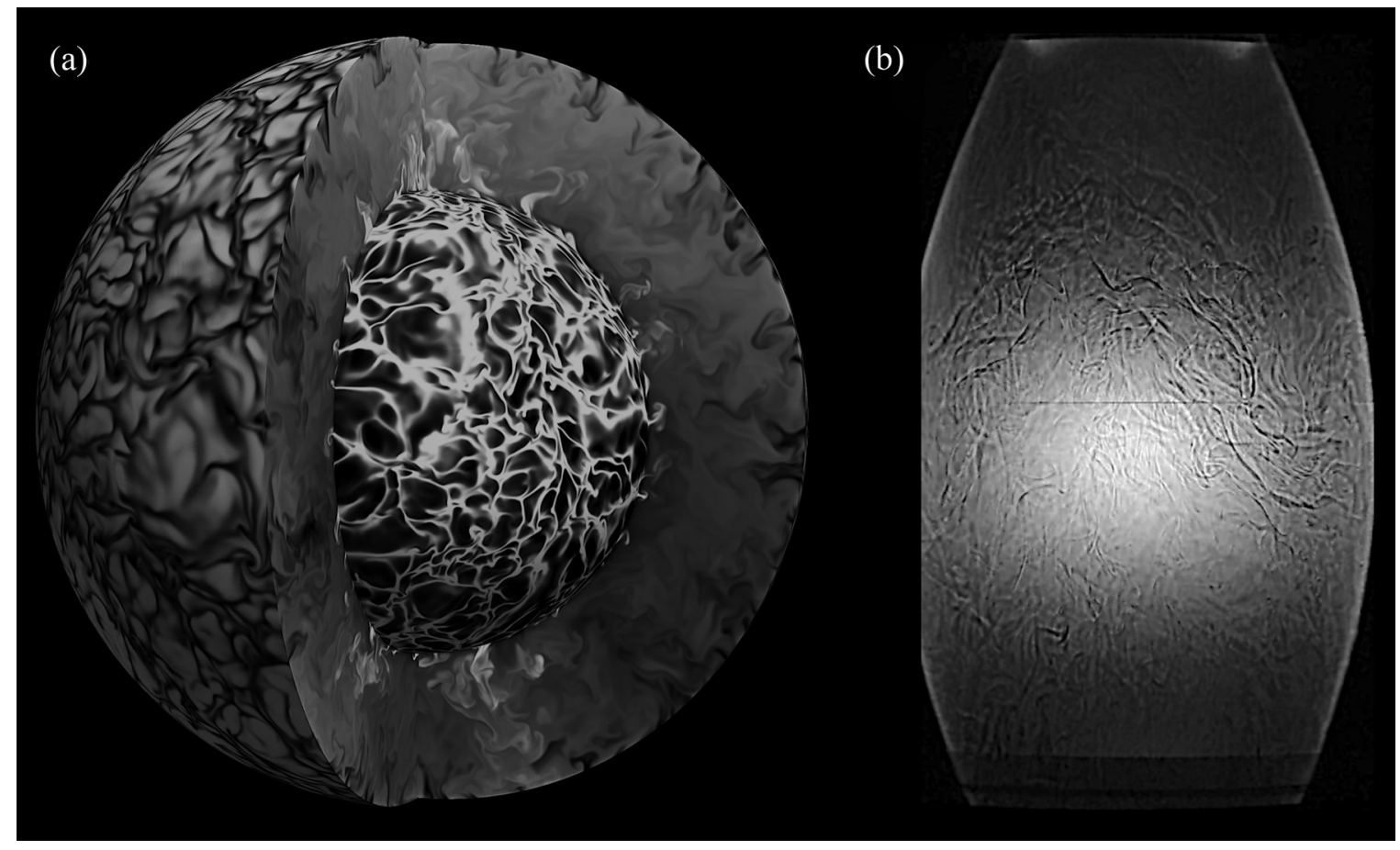

FIG. 2. Nonrotating convective flows, which approximate the slowly rotating limit (SRL). (a) Temperature field image adapted from the $\mathrm{Ra}=10^{8} ; \operatorname{Pr}=1$; radius ratio $\chi=0.6$ spherical shell simulation of Gastine et al. [67], corresponding to $\operatorname{Re}_{f f}=10^{4}$. Lighter (darker) shading represents warmer (cooler) fluid. (b) Laboratory shadowgraph image courtesy of Jewel Abbate (UCLA) showing convection in 1.5 cSt silicone oil for $\mathrm{Ra} \simeq 4 \times 10^{11}$ and $\operatorname{Pr} \simeq 21$, corresponding to $\operatorname{Re}_{f f} \simeq 10^{5}$. The cylindrical tank is $40 \mathrm{~cm}$ high by $20 \mathrm{~cm}$ across, with shape distorted and its left-hand side clipped by the shadowgraph technique. The horizontal line near the midplane and the dark region at the tank bottom are further lighting artifacts.

the RRL [55]. With this guiding principle in mind, the Ekman number is typically fixed at some low value whilst $\mathrm{Ra}$ is varied along each data shingle. Figure 1(b), which is adapted from the laboratory-based study of Cheng et al. [52], shows this approach well. Three different fixed Ekman number shingles are shown. Rayleigh number values are shown on the $x$ axis and the $y$ axis denotes the Nusselt number values. [The solid black line denotes the NRL scaling $\mathrm{Nu}_{0}(\mathrm{Ra})$.] Small $x$ 's on the abscissa denote $\mathrm{Ra}_{\text {Crit }}=8.7 \mathrm{Ek}^{-4 / 3}$, the critical Ra value at which stationary planar rapidly rotating convection onsets at a given Ek value. Such a survey uses $\widetilde{R a}=\mathrm{Ra} / \mathrm{Ra}_{\text {crit }}=1$ as its philosophical starting point, and then perturbs the system with ever-increasing values of $\widetilde{R a}$. In these studies, $\mathrm{Ro}_{C}$ is not used as a control parameter, but is often checked a posteriori to see if it can collapse the data $[22,46,52,56]$.

The two panels of Fig. 1 are qualitative mirror images of one another. Starting from different ends of the inertially versus rotationally dominated ranges, they show nearly identical data but harvested along different slices through the same parameter spaces. Figure 1(a) assumes a high Ra, SRL dominated by buoyancy effects, whereas Fig. 1(b) assumes a low Ek, RRL dominated by Coriolis forces.

The goal of this paper is to develop transport scalings that bridge the gaps between the NRL, SRL, and RRL convective world views. A particularly important finding is the relative importance of the free-fall terminal velocity in the NRLs and SRLs and of the thermal wind terminal velocity in the RRL, and how these velocities are related to one another via $\operatorname{Ro}_{C}$.

\section{THE NONROTATING AND SLOWLY ROTATING LIMITS}

In the limit of asymptotically high Ra, high Re, turbulent convection, we presume that perfect power-law scaling behaviors exist to describe the heat and momentum transport in terms of the other relevant system parameters, $\mathrm{Nu}(\mathrm{Ra}, \mathrm{Pr})$ and $\mathrm{Re}(\mathrm{Ra}, \mathrm{Pr})$ [43,57-59]. The demonstration of such asymptotic scalings is still an active and frothy topic of scientific debate [60-65]. We assume, further, that similar transport scalings exist in the nonrotating and slowly rotating regimes. Despite small differences due to symmetry breaking in slowly rotating systems $[44,66]$, their gross transport behaviors can be taken to be comparable (e.g., Fig. 2).

For both nonrotating and slowly rotating convections, we take the characteristic convection length scale to be the global scale of the system in all directions, $\ell \sim H$, based on the superstructures that form at high Ra with vertical scales of order $H$ and lateral scales that are typically less than $10 H$ [68-72], which appear to be maintained even in extreme astrophysical and geophysical systems [73]. In the turbulent limit, the free-fall inertial balance is achieved:

$$
\boldsymbol{u} \cdot \nabla \boldsymbol{u} \sim \boldsymbol{g} \alpha \theta \longrightarrow \frac{U^{2}}{H} \sim g \alpha \vartheta .
$$

Analytic estimations for the characteristic magnitude of $\vartheta$ in the turbulent regime are nontrivial $[1,59]$. Here, following the work of Grossmann and Lohse [74], we scale $\vartheta \sim \Delta T$. In the 
NRL and SRL, it then follows that

$$
\ell \sim H, \vartheta \sim \Delta T \text { and } U \sim \sqrt{g \alpha \Delta T H} \equiv U_{f f} .
$$

The dominant flows in these regimes are large scale; they are driven by thermal fluctuations that are roughly comparable to the temperature drop across the system (likely akin to the characteristic boundary layer temperature variations); and the convective flows will approach $U_{f f}$, the diffusivity-free, inertial free-fall velocity $[58,75,76]$. Further, the characteristic advective timescales are isotropic and are given by

$$
\tau_{U}=\frac{\ell}{U} \sim \frac{H}{U_{f f}}=\sqrt{H /(g \alpha \Delta T)} \equiv \tau_{f f},
$$

where $\tau_{f f}$ is the inertial free-fall time across the system. We note, following Spiegel [75], that our assumption that transport processes are dominated by the large scale flows likely best applies in low Pr fluids [71]. We will not probe this assumption more deeply here, but direct readers to more focused treatments of nonrotating Rayleigh-Bénard convection $[1,59,77,78]$.

Using the SRL scales given in (17), the local Rossby number can be recast as

$$
\operatorname{Ro}_{\ell}=\frac{U}{2 \Omega \ell} \sim \frac{U_{f f}}{2 \Omega H}=\sqrt{\frac{g \alpha \Delta T}{4 \Omega^{2} H}} \equiv \operatorname{Ro}_{C},
$$

which demonstrates that the local Rossby number, $\mathrm{Ro}_{\ell} \gg$ 1 , is equivalent to the convective Rossby number, $\mathrm{Ro}_{C}$, in the SRL. Further, from Eq. (19), we arrive at the standard, timescale-based description of the convective Rossby number, $\operatorname{Ro}_{C}=\tau_{\Omega} / \tau_{f f}$, as the ratio of the rotational time, $\tau_{\Omega}=$ $1 /(2 \Omega)$, and the free-fall time across the system scale.

The scales in Eq. (17) lead to the following NRL and SRL transport estimates:

$$
\begin{aligned}
\operatorname{Re}_{\ell}=\frac{U \ell}{v} \sim \frac{U_{f f} H}{v} & =\frac{\sqrt{g \alpha \Delta T H^{3}}}{v} \\
& =\left(\frac{\mathrm{Ra}}{\operatorname{Pr}}\right)^{1 / 2} \equiv \operatorname{Re}_{f f}, \\
\mathrm{Pe}_{\ell}=\frac{U \ell}{\kappa} \sim \frac{U_{f f} H}{\kappa} & =\frac{\sqrt{g \alpha \Delta T H^{3}}}{\kappa} \\
& =(\operatorname{RaPr})^{1 / 2} \equiv \operatorname{Pe}_{f f}, \\
\mathrm{Nu} \sim \frac{U \vartheta H}{\kappa \Delta T} \sim \frac{U_{f f} H}{\kappa} & =(\operatorname{RaPr})^{1 / 2} \equiv \operatorname{Pe}_{f f} .
\end{aligned}
$$

Dimensional analysis can be used, independently, to solve for the exponents $\zeta$ and $\chi$ that yield diffusivity-free expressions for the characteristic transport parameters [4], yielding

$$
\begin{aligned}
\operatorname{Re}_{H} \sim \widetilde{\operatorname{Ra}}^{\zeta} \operatorname{Pr}= & (\operatorname{Ra} / \operatorname{Pr})^{1 / 2} \equiv \operatorname{Re}_{f f} \\
& (\zeta=-\chi=1 / 2), \\
\operatorname{Pe}_{H} \sim \widetilde{\operatorname{Ra}}^{\zeta} \operatorname{Pr}^{\chi}= & (\operatorname{RaPr})^{1 / 2} \equiv \operatorname{Pe}_{f f} \\
& (\zeta=\chi=1 / 2), \\
\mathrm{Nu} \sim \widetilde{\operatorname{Ra}}^{\zeta} \operatorname{Pr}^{\chi}= & (\operatorname{RaPr})^{1 / 2} \equiv \operatorname{Pe}_{f f} \\
& (\zeta=\chi=1 / 2),
\end{aligned}
$$

where $\widetilde{\mathrm{Ra}} \mapsto \mathrm{Ra}$ in the dimensional analysis, since $\mathrm{Ra}_{\text {crit }}$ is effectively constant in the nonrotating and SRLs. Because it is being assumed that the convection is highly supercritical and turbulence dominated, we take $(\mathrm{Nu}-1) \approx \mathrm{Nu},(\mathrm{Re}-1) \approx$ $\mathrm{Re}$, and $(\widetilde{\mathrm{Ra}}-1) \approx \widetilde{\mathrm{Ra}}$ in all our dimensional analyses, cf. Ref. [79].

The dimensional analytical transport estimates in Eqs. (21) are consistent with the dynamical scaling estimates given in Eqs. (20) and also agree with the classic dimensional analysis predictions for nonrotating convection in the limit of zero diffusive effects [76]. The agreement between the independent scalings Eqs. (20) and (21) shows that $\operatorname{Re}_{\ell} \sim \operatorname{Re}_{H}$ and $\mathrm{Pe}_{\ell} \sim \mathrm{Pe}_{H}$, consistent with our assumption that $\ell \sim H$ in NRL and SRL. Lastly, multiplying by Ek, the momentum transport scalings Eqs. (20a) and (21a) require that

$$
\mathrm{Ro}_{\ell} \sim \operatorname{Ro}_{H} \sim \operatorname{Ro}_{C}
$$

in the slowly rotating regime, consistent with Eq. (19).

\section{THE RAPIDLY ROTATING LIMIT}

Just as angular momentum is the key dynamical variable in rapidly rotating solid mechanics problems, vorticity, $\omega=$ $\boldsymbol{\nabla} \times \boldsymbol{u}$, is the essential dynamical variable in rapidly rotating fluid systems in which rotational inertia dominates the physics [80]. The evolution equation for fluid vorticity, $\nabla \times(1 \mathrm{a})$, is

$$
\begin{aligned}
\partial_{t} \boldsymbol{\omega}+\boldsymbol{u} \cdot \nabla \boldsymbol{\omega}-\boldsymbol{\omega} \cdot \nabla \boldsymbol{u}= & 2 \boldsymbol{\Omega} \cdot \nabla \boldsymbol{u} \\
& +\nabla \times(\boldsymbol{g} \alpha \theta)+v \nabla^{2} \boldsymbol{\omega} .
\end{aligned}
$$

In the turbulent RRL, a balance is achieved in Eq. (23) between the inertial (I), Coriolis (C), and buoyancy (A, for Archimedean) terms [81,82]. This is typically referred to as the CIA balance $[2,83]$,

$$
\begin{aligned}
\boldsymbol{u} \cdot \nabla \omega & \sim 2 \Omega \partial_{z} \boldsymbol{u} \sim \nabla \times(\boldsymbol{g} \alpha \theta) \\
\frac{U^{2}}{\ell^{2}} & \sim \frac{2 \Omega U}{H} \sim \frac{g \alpha \vartheta}{\ell},
\end{aligned}
$$

in which the first term is inertial advection of vorticity (I), the second is the axial stretching of planetary (or background) vorticity (C), and the third is the buoyancy torque (A).

Rapidly rotating convective motions are strongly anisotropic, as shown in Fig. 3, with small scales perpendicular to $\boldsymbol{\Omega}$ and much longer scales parallel to $\boldsymbol{\Omega}$. Therefore, it is essential in (24) to distinguish between the characteristic convection scale $\ell$ measured perpendicular to $\boldsymbol{\Omega}$ and the system scale $H$ measured parallel $\boldsymbol{\Omega}$. Only the stretching of the background vorticity, $2 \Omega \partial_{z} \boldsymbol{u}$, can occur on the system scale. The other two terms, I and A, operate on the local convective scale. Although the length scales $\ell$ and $H$ differ greatly in rapidly rotating convection, the kinetic energies measured along these different directions remain comparable, even in the supercritical regime $[17,50,85,86]$. Thus, we assume that the characteristic velocity magnitudes are approximately isotropic $\left|u_{i}\right| \sim U$ in RRL.

The balance between the $\mathrm{C}$ and I terms in (24) then gives

$$
\frac{\ell}{H} \sim \frac{U}{2 \Omega \ell} \equiv \operatorname{Ro}_{\ell}=\frac{\tau_{\Omega}}{\tau_{U}^{\ell}},
$$




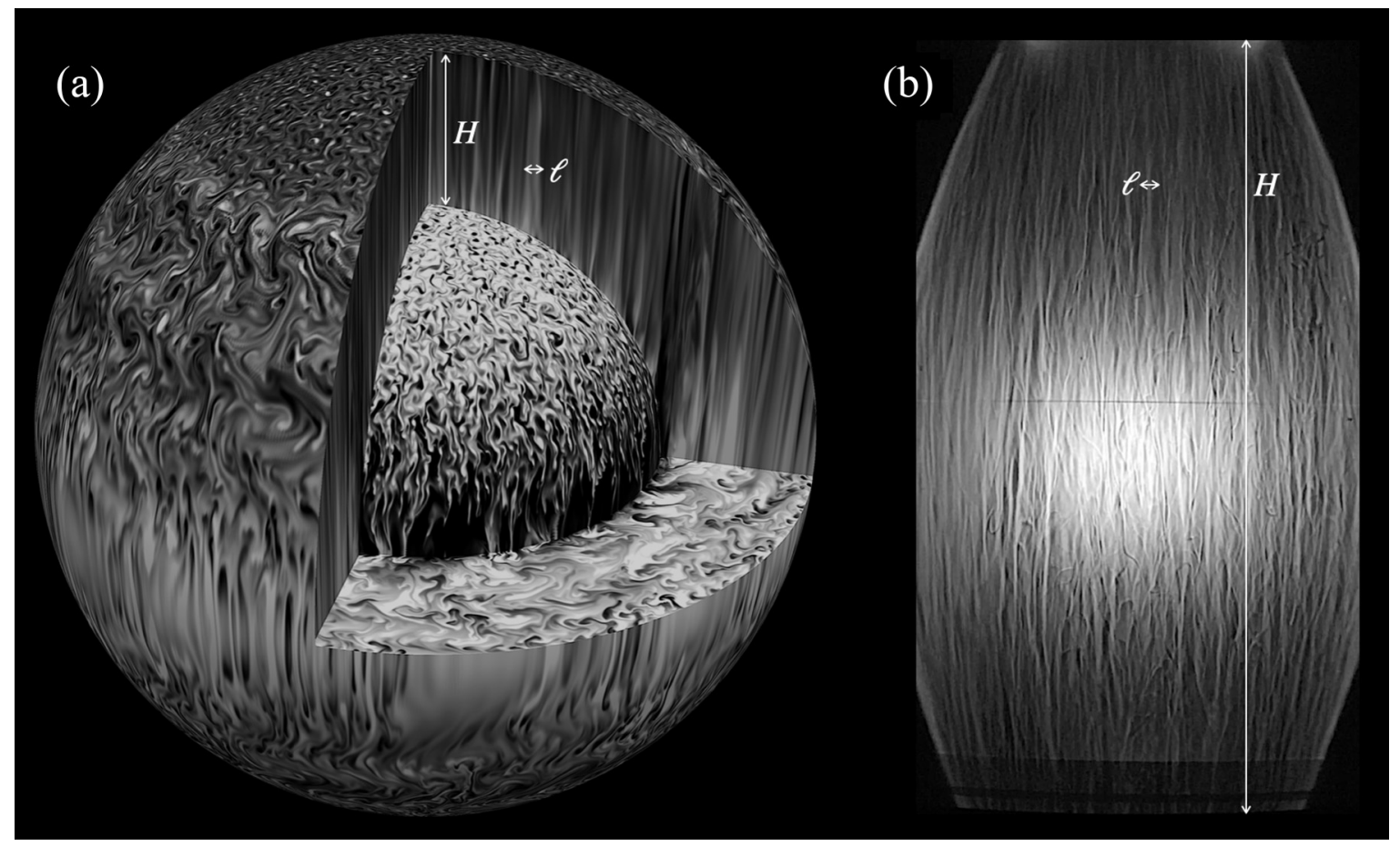

FIG. 3. Anisotropic flows in rapidly rotating convection with longer characteristic axial scales than horizontal scales $\ell$ (measured perpendicular to the rotation axis). (a) Temperature field image from the $\mathrm{Ra}=2.5 \times 10^{9} ; \mathrm{Ek}=10^{-6} ; \operatorname{Pr}=1$; radius ratio $\chi=0.6$ spherical shell simulation of Gastine et al. [84], corresponding to $\operatorname{Ro}_{C}=5 \times 10^{-2}$ and $\operatorname{Re}_{\mathrm{TW}} \simeq 1.3 \times 10^{2}$. Lighter (darker) shading represents warmer (cooler) fluid. (b) Laboratory shadowgraph image courtesy of Jewel Abbate (UCLA) showing rotating convection in $1.5 \mathrm{cSt}$ silicone oil for $\mathrm{Ra} \simeq 5 \times 10^{11} ; \mathrm{Ek} \simeq 6 \times 10^{-7} ; \mathrm{Pr} \simeq 21$, corresponding to $\operatorname{Ro}_{C} \simeq 9 \times 10^{-2}$ and $\operatorname{Re}_{\mathrm{TW}} \simeq 1.3 \times 10^{3}$. The cylindrical tank is $40 \mathrm{~cm}$ high by $20 \mathrm{~cm}$ across, with its shape distorted and clipped around the midplane by the shadowgraph imaging technique. The horizontal line near the midplane and the dark region at the tank bottom are further lighting artifacts.

where the lateral advective timescale $\tau_{U}^{\ell}=\ell / U$ characterizes rapidly rotating convection. Thus, rapidly rotating convection is highly anisotropic with $\ell \ll H$, since $\ell \sim \operatorname{Ro}_{\ell} H$ in (25) and $\mathrm{Ro}_{\ell} \ll 1$ in the definition of the RRL. Unlike in the NRL and SRL, where the bulk fluid tends to be isothermalized by strong turbulence, in rapidly rotating convection, an unstable mean temperature gradient tends to be sustained in the fluid bulk, $\partial_{g} \bar{T} \sim \Delta T / H[40,52,86,87]$. The fluctuating thermal energy Eq. (1b) thus scales as

$$
(\boldsymbol{u} \cdot \nabla \theta)^{\prime} \sim\left(\hat{\boldsymbol{e}}_{\boldsymbol{g}} \cdot \boldsymbol{u}\right) \partial_{g} \bar{T} \longrightarrow \frac{U \vartheta}{\ell} \sim \frac{U \Delta T}{H} .
$$

This implies, in the RRL, that

$$
\frac{\vartheta}{\Delta T} \sim \frac{\ell}{H} \sim \mathrm{Ro}_{\ell} .
$$

Balancing the $\mathrm{C}$ and $\mathrm{A}$ terms in (24) yields

$$
U \sim \frac{g \alpha \Delta T}{2 \Omega}\left(\frac{\vartheta}{\Delta T} \frac{H}{\ell}\right) \sim \frac{g \alpha \Delta T}{2 \Omega} \equiv U_{\mathrm{TW}},
$$

where $U_{\mathrm{TW}}$, the thermal wind velocity, is the diffusivity-free velocity scale in the rapidly rotating convection regime [88]. (This thermal wind scaling is similarly found by balancing the I and A terms in (24).) From (28), we see that the local advection time scale in RRL is the thermal wind timescale:

$$
\tau_{U}^{\ell}=\ell / U_{T W} \equiv \tau_{T W} .
$$

The rapidly rotating local Rossby number then becomes

$$
\begin{aligned}
\mathrm{Ro}_{\ell} \sim \frac{U_{\mathrm{TW}}}{2 \Omega \ell} & =\frac{\tau_{\Omega}}{\tau_{\mathrm{TW}}}=\frac{g \alpha \Delta T}{(2 \Omega)^{2} H} \frac{1}{\mathrm{Ro}_{\ell}} \\
& \longrightarrow \mathrm{Ro}_{\ell} \sim \sqrt{\frac{\mathrm{RaEk}^{2}}{\mathrm{Pr}}} \equiv \mathrm{Ro}_{C} .
\end{aligned}
$$

Thus, the a posteriori local Rossby number, $\mathrm{Ro}_{\ell}$, is equivalent to the a priori convective Rossby number, $\mathrm{Ro}_{C}$, in both the SRL Eq. (19) and in the RRL Eq. (30). At closer inspection, this holds because the local advective timescales, $\tau_{f f}=H / U_{f f}$ in SRL and $\tau_{\mathrm{TW}}=\ell / U_{\mathrm{TW}}$ in RRL, are similar. Thus, their ratio yields

$$
\frac{\tau_{f f}}{\tau_{\mathrm{TW}}} \sim \frac{H}{\ell} \frac{U_{\mathrm{TW}}}{U_{f f}} \sim \frac{1}{\operatorname{Ro}_{C}} \frac{g \alpha \Delta T /(2 \Omega)}{\sqrt{g \alpha \Delta T H}}=\mathcal{O}(1) .
$$

This similarity between the SRL and the RRL local advective timescales explains why the convective Rossby number turns up so ubiquitously in rotating convection dynamics: Even though $U_{f f}$ and $H$ in SRL both greatly exceed $U_{\mathrm{TW}}$ and $\ell$ in RRL, their ratios, $U_{f f} / H$ and $U_{\mathrm{TW}} / \ell$ have equivalent scaled values. Expression (31) demonstrates, further, that the convective Rossby number can be cast, alternatively, as

$$
\operatorname{Ro}_{C} \equiv \frac{U_{\mathrm{TW}}}{U_{f f}} .
$$

This velocity-based definition of $\mathrm{Ro}_{C}$ holds in both slowly rotating and rapidly rotating regimes, and differs in its 
interpretation in comparison to the standard (slowly rotating) definition in which $\operatorname{Ro}_{C}=\tau_{\Omega} / \tau_{f f}$, as will be discussed further in Sec. VI.

In the limit of rapidly rotating convective turbulence, the CIA balance gives

$$
\begin{gathered}
\ell \sim \operatorname{Ro}_{C} H, \quad \vartheta \sim \operatorname{Ro}_{C} \Delta T, \\
U \sim \operatorname{Ro}_{C} U_{f f}=\frac{g \alpha \Delta T}{2 \Omega} \equiv U_{\mathrm{TW}},
\end{gathered}
$$

with all three turbulent RRL scales differing by $\mathrm{Ro}_{C}$ relative to their corresponding SRL scales. Following the same steps as in Eq. (17) but employing the rapidly rotating scales in Eq. (34) then leads to the following RRL transport estimates:

$$
\begin{aligned}
\operatorname{Re}_{\ell} & =\frac{U_{\mathrm{TW}} \ell}{v} \sim \frac{g \alpha \Delta T}{2 \Omega} \frac{\ell}{v}=\left(\frac{\mathrm{Ra}}{\mathrm{Pr}}\right)^{3 / 2} \mathrm{Ek}^{2} \\
& =\operatorname{Ro}_{C}^{2} \operatorname{Re}_{f f} \equiv \mathrm{Re}_{\mathrm{TW}} \\
\mathrm{Pe}_{\ell} & =\frac{U_{\mathrm{TW}} \ell}{\kappa} \sim \frac{g \alpha \Delta T}{2 \Omega} \frac{\ell}{\kappa}=\left(\frac{\mathrm{Ra}^{3 / 2}}{\mathrm{Pr}^{1 / 2}}\right) \mathrm{Ek}^{2} \\
& =\operatorname{Ro}_{C}^{2} \mathrm{Pe}_{f f} \equiv \mathrm{Pe}_{\mathrm{TW}} \\
\mathrm{Nu} \sim \frac{U_{\mathrm{TW}}}{\kappa}\left(\frac{\vartheta H}{\Delta T}\right) & =\frac{U_{\mathrm{TW}} \ell}{\kappa}=\left(\frac{\mathrm{Ra}^{3 / 2}}{\mathrm{Pr}^{1 / 2}}\right) \mathrm{Ek}^{2} \\
& =\operatorname{Ro}_{C}{ }^{2} \mathrm{Pe}_{f f} \equiv \mathrm{Pe}_{\mathrm{TW}},
\end{aligned}
$$

where $\mathrm{Re}_{\mathrm{TW}}$ and $\mathrm{Pe}_{\mathrm{TW}}$ are the thermal wind Reynolds and thermal wind Péclet numbers, respectively.

The scaling analysis in Eq. (35) is consistent with rapidly rotating, diffusivity-free dimensional analysis, which yields

$$
\begin{aligned}
& \operatorname{Re}_{\ell} \sim \widetilde{\operatorname{Ra}^{\zeta}} \operatorname{Pr}^{\chi}=(\operatorname{Ra} / \operatorname{Pr})^{3 / 2} \mathrm{Ek}^{2} \equiv \operatorname{Re}_{\mathrm{TW}} \\
& (\zeta=-\chi=3 / 2) \text {, } \\
& \mathrm{Pe}_{\ell} \sim \widetilde{\operatorname{Ra}}^{\zeta} \operatorname{Pr}^{\chi}=\left(\frac{\mathrm{Ra}^{3 / 2}}{\operatorname{Pr}^{1 / 2}}\right) \mathrm{Ek}^{2} \equiv \mathrm{Pe}_{\mathrm{TW}} \\
& (\zeta=-3 \chi=3 / 2) \text {, } \\
& \mathrm{Nu} \sim \widetilde{\operatorname{Ra}}^{\zeta} \operatorname{Pr}^{\chi}=\left(\frac{\mathrm{Ra}^{3 / 2}}{\operatorname{Pr}^{1 / 2}}\right) \mathrm{Ek}^{2} \equiv \mathrm{Pe}_{\mathrm{TW}} \\
& (\zeta=-3 \chi=3 / 2),
\end{aligned}
$$

where the critical Rayleigh number varies strongly here with the system's rotation, $\widetilde{\mathrm{Ra}} \sim \mathrm{RaEk}^{4 / 3}$. Consistency between Eq. (35) and (36) requires that the pertinent velocity and length scales must be $U_{\mathrm{TW}}$ and $\ell$ in RRL. Thus, $\operatorname{Re} \sim \operatorname{Re}_{\ell} \equiv \operatorname{Re}_{\mathrm{TW}}$ and $\mathrm{Pe} \sim \mathrm{Pe}_{\ell} \equiv \mathrm{Pe}_{\mathrm{TW}}$ in the rapidly rotating regime. Multiplying Eq. (35a) by the local Ekman number, $\mathrm{Ek}_{\ell}=v /\left(2 \Omega \ell^{2}\right)$, yields $\operatorname{Ro}_{\ell}=\mathrm{Re}_{\ell} \mathrm{Ek}_{\ell} \sim \mathrm{Ro}_{C}$, consistent with Eq. (30). Further, the RRL heat transport scaling Eq. (35c) is also consistent with asympotically reduced theory and diffusivity-free formulations [4,89-91]. Recent studies, such as Plumley et al. [92,93], suggest that it is possible to reach the RRL scalings Eq. (35) at far lower Ra values than are necessary to reach their diffusivity-free nonrotating counterparts, cf. Ref. [78].

The rapidly rotating thermal wind transport scalings in Eq. (35) differ from the slowly rotating free-fall scalings by a factor of $\operatorname{Ro}_{C}{ }^{2}$. This creates a clean and novel link between the two sets of scaling predictions. We can alternatively cast the RRL expressions as

$$
\begin{aligned}
& \operatorname{Re}_{\ell} \sim \operatorname{Ro}_{C}^{3} \mathrm{Ek}^{-1}, \\
& \mathrm{Pe}_{\ell} \sim \mathrm{Nu} \sim \operatorname{Ro}_{C}^{3}(\mathrm{Ek} / \mathrm{Pr})^{-1} .
\end{aligned}
$$

From (24), we predict that rapidly rotating turbulent transport data acquired with approximately fixed rotation rate and material properties will be collapsed when normalized by the cube of the convective Rossby number.

Local scale parametrizations naturally arise in our analysis of rapidly rotating transport phenomena. However, the system-scale transport parameters, $\mathrm{Re}_{H}$ and $\mathrm{Pe}_{H}$, are most often reported in the literature [30]. Thus, we rescale our local rapidly rotating transport scalings to provide the equivalent, system-scale counterparts:

$$
\begin{aligned}
\operatorname{Re}_{H} & =\operatorname{Re}_{\ell} \frac{H}{\ell} \sim \frac{U_{\mathrm{Tw}} H}{v}=\frac{\mathrm{RaEk}}{\operatorname{Pr}} \\
& =\operatorname{Ro}_{C}{ }^{-1} \operatorname{Re}_{\mathrm{TW}}=\operatorname{Ro}_{C} \operatorname{Re}_{f f} \\
\mathrm{Pe}_{H} & =\operatorname{Pe}_{\ell} \frac{H}{\ell} \sim \frac{U_{\mathrm{TW}} H}{\kappa}=\mathrm{RaEk} \\
& =\operatorname{Ro}_{C}{ }^{-1} \mathrm{Pe}_{\mathrm{TW}}=\operatorname{Ro}_{C} \mathrm{Pe}_{f f} .
\end{aligned}
$$

In addition, the system-scale Rossby number scales as

$$
\operatorname{Ro}_{H}=\operatorname{Re}_{H} \mathrm{Ek} \sim \operatorname{Ro}_{C}{ }^{2},
$$

in agreement with the low-Ek, quasigeostrophic convection models of Guervilly et al. [94] and the three-dimensional asymptotically-reduced models of Maffei et al. [91]. This system-scale RRL Rossby number scaling (39) differs by a factor of $\operatorname{Ro}_{C}$ relative to the slowly rotating scaling (22) in which $\operatorname{Ro}_{H} \sim \operatorname{Ro}_{\ell} \sim \operatorname{Ro}_{C}$.

\section{FLUX-BASED SCALINGS}

\section{A. Nonrotating and slowly rotating flux-based scalings}

When considering a planetary or stellar convection system, it is far easier to estimate the outward thermal flux than to infer a temperature drop across a given fluid layer. Therefore, it is of great utility to recast the scalings developed above in terms of the (superadiabatic) heat flux, $Q$, instead of the temperature difference, $\Delta T$. Nondimensionally, this simply corresponds to replacing the Rayleigh number, $\mathrm{Ra} \propto \Delta T$, with the flux Rayleigh number, $\mathrm{Ra}_{F}=\mathrm{RaNu} \propto Q$. To recast the NRL and SRL scalings in terms of $\mathrm{Ra}_{F}$, we manipulate Eq. (20c) into the form

$$
\mathrm{Ra} \sim\left[\operatorname{RaNuPr}^{-1 / 2}\right]^{2 / 3} \sim \operatorname{Ra}_{F}^{2 / 3} \operatorname{Pr}^{-1 / 3},
$$

and substitute this into Eqs. (20a) and (20b), giving the fluxbased free-fall scalings

$$
\operatorname{Re}_{f f} \sim\left[\frac{\operatorname{Ra}_{F}}{\operatorname{Pr}^{2}}\right]^{1 / 3} \text { and } \operatorname{Pe}_{f f} \sim\left[\operatorname{Ra}_{F} \operatorname{Pr}\right]^{1 / 3} .
$$

The SRL flux-based expression for the Rossby number is then [26]

$$
\operatorname{Ro}_{\ell} \sim \operatorname{Ro}_{H} \sim\left[\frac{\mathrm{Ra}_{F} \mathrm{Ek}^{3}}{\operatorname{Pr}^{2}}\right]^{1 / 3}=\operatorname{Ra}_{F}^{* 1 / 3}
$$


The respective dimensional forms of the length, uncontrolled temperature drop (which is assumed here to be proportional to $\vartheta$ in NRL and SRL) and free-fall velocity scale in the slowly rotating regime are

$$
\begin{aligned}
\ell & \sim H, \\
\Delta T & \sim Q / \rho c_{P} U_{f f} \sim\left(\frac{Q^{2}}{g \alpha \rho^{2} c_{P}^{2} H}\right)^{1 / 3}, \\
U & \sim U_{f f} \sim \sqrt{g \alpha \Delta T H} \sim\left[\frac{g \alpha Q H}{\rho c_{P}}\right]^{1 / 3} .
\end{aligned}
$$

Equations (43) correspond to the free-fall balance expressed in terms of an applied heat flux $Q$ [95]. Further, by inserting Eq. (43b) into Eq. (15), we find that the flux-based SRL expression for $\mathrm{Ro}_{C}=\mathrm{Ra}_{F}^{* 1 / 3}$, which is identical to $\mathrm{Ro}_{\ell}$ in (42). Thus, $\mathrm{Ro}_{\ell} \approx \mathrm{Ro}_{C}$ in the flux-based framework as well, as must be the case since this result is framework independent.

\section{B. Rapidly rotating flux-based scalings}

To formulate the flux-based, system-scale, rapidly rotating momentum transport scaling, we recast the RRL heat transport scaling (35c) as

$$
\mathrm{Ra}=\left(\operatorname{RaNuPr}^{1 / 2} \mathrm{Ek}^{-2}\right)^{2 / 5}=\operatorname{Ra}_{F}^{2 / 5} \operatorname{Pr}^{1 / 5} \mathrm{Ek}^{-4 / 5} .
$$

Substituting Eq. (44) into Eq. (35) leads to the local, fluxbased, rapidly rotating transport scalings:

$\mathrm{Re}_{\mathrm{TW}} \sim\left[\frac{\mathrm{Ra}_{F} \mathrm{Ek}^{4 / 3}}{\operatorname{Pr}^{2}}\right]^{3 / 5}=\mathrm{Ra}_{F}^{* 3 / 5} \mathrm{Ek}^{-1}=\mathrm{Ro}_{C}{ }^{3} \mathrm{Ek}^{-1}$,

$\mathrm{Pe}_{\mathrm{TW}} \sim\left[\frac{\mathrm{Ra}_{F} \mathrm{Ek}^{4 / 3}}{\operatorname{Pr}^{1 / 3}}\right]^{3 / 5}=\operatorname{Ra}_{F}^{* 3 / 5}(/ \operatorname{Pr})^{-1}=\operatorname{Ro}_{C}{ }^{3}(\mathrm{Ek} / \mathrm{Pr})^{-1}$,

with the local scale, RRL flux-based Rossby number

$$
\mathrm{Ro}_{\ell} \sim\left[\frac{\mathrm{Ra}_{F} \mathrm{Ek}^{3}}{\operatorname{Pr}^{2}}\right]^{1 / 5}=\mathrm{Ra}_{F}^{* 1 / 5}=\operatorname{Ro}_{C} .
$$

Note, using the flux-based expression for $U_{\mathrm{TW}}$ [given in Eqs. (50) below], one finds again that $\mathrm{Ro}_{\ell} \approx \mathrm{Ro}_{C}$. However, in contrast to the fixed temperature configuration, the fluxbased local Rossby numbers, Eq. (42) in SRL and Eq. (46) in RRL, are no longer identical. Instead, both flux-based $\mathrm{Ro}_{\ell}$ expressions depend on the modified flux Rayleigh number, but in the SRL $\mathrm{Ra}_{F}^{*}$ is raised to the one-third power, whereas it is raised to the one-fifth power in the RRL. This difference in the flux-based $\mathrm{Ro}_{\ell}$ expressions stems from the different $\mathrm{Ra}\left(\mathrm{Ra}_{F}\right.$, Ek, Pr) scalings given in Eqs. (40) and (44).

The system-scale, flux-based, rapidly rotating transport scalings are often used in the geophysical and astrophysical literature $[23,96]$. These are found by substituting Eq. (44) into Eq. (38a), which leads to

$$
\begin{aligned}
\operatorname{Re}_{H} \sim\left[\frac{\operatorname{Ra}_{F}}{\operatorname{Pr}^{2}}\right]^{2 / 5} \mathrm{Ek}^{1 / 5} \equiv \operatorname{Re}_{\mathrm{CIA}}, \\
\operatorname{Pe}_{H} \sim \operatorname{Ra}_{F}^{2 / 5}(\mathrm{Ek} \operatorname{Pr})^{1 / 5}=\operatorname{Re}_{\mathrm{CIA}} \operatorname{Pr} .
\end{aligned}
$$

The flux-based $\mathrm{Re}_{H}$ expression Eq. (47a) is referred to as the CIA scaling velocity, $\mathrm{Re}_{\mathrm{CIA}}$, since it is indeed derived from the CIA triple balance [2,81-83]. This flux-based momentum transport scaling is easily converted back into a temperaturebased scaling by substituting $\mathrm{Ra}_{F}=\mathrm{RaNu}$ into $\mathrm{Re}_{\mathrm{CIA}}$ and then further substituting in $\mathrm{Nu} \sim \mathrm{Ra}^{3 / 2} \mathrm{Ek}^{2} / \mathrm{Pr}^{1 / 2}=\mathrm{Pe}_{\mathrm{TW}}$. Doing so yields

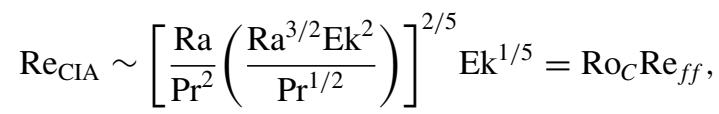

in agreement with Eqs. (35a) and (38a). Multiplying (48) by Ek then demonstrates that

$$
\operatorname{Ro}_{\text {CIA }} \sim \operatorname{Ro}_{C}{ }^{2} \quad\left(\text { when } \mathrm{Nu} \rightarrow \mathrm{Pe}_{\mathrm{TW}}\right) .
$$

This shows that the classical, flux-based CIA theory is synonymous with the temperature-based rapidly rotating velocity scalings given in Eqs. (38a) and (39).

Since most laboratory and numerical simulations cannot reach the diffusivity-free $\mathrm{Pe}_{\mathrm{TW}}$ heat transfer trend, the $R o_{H} \sim$ $\operatorname{Ro}_{C}{ }^{2}$ scaling is difficult to attain, cf. Refs. [91,94,97]. For example, in the seminal planetary dynamo survey of Christensen and Aubert [25], it was found that $\mathrm{Ro}_{H} \sim \mathrm{Ra}_{F}^{* 2 / 5}$, which, comparing to Eq. (47a), shows that the bulk flow had attained the turbulent CIA scaling. Their heat transfer data was best fit as $\mathrm{Nu} \sim \mathrm{RaEk}$, which differs from the $\mathrm{Pe}_{\mathrm{TW}}$ scaling likely because it was controlled by diffusive, boundary layer physics [54,98]. This corresponds to $R o_{H} \sim \operatorname{Ro}_{C}{ }^{8 / 5}$. However, if we substitute $\mathrm{Nu}=\mathrm{Ra}^{3 / 2} \mathrm{Ek}^{2} / \mathrm{Pr}^{1 / 2}$ in place of their $\mathrm{Nu} \sim \mathrm{RaEk}$ scaling, then the system-scale Rossby number scaling necessarily transforms to $\mathrm{Ro}_{H} \sim \mathrm{Ro}_{C}{ }^{2}$.

Our flux-based momentum transport scalings help to tie the proverbial room together by showing that the RRL transport Eq. (38a) is formally identical to the classical, flux-based CIA velocity scaling Eq. (47a) when $\mathrm{Nu} \approx \mathrm{Pe}_{\mathrm{Tw}}$. However, this $\mathrm{Pe}_{\mathrm{TW}}$ heat transfer scaling is not often found in standard experiments or direct numerical simulations, because the heat transfer rarely reaches the RRL trend $[89,90,92,93]$. This is an important physical point, as the flux-based $\mathrm{Re}_{H}$ scaling in Eq. (47a) can be applied for any $\mathrm{Nu}$ value and, accordingly, is often considered to be fundamentally different from, and to conflict with, the local scale prediction Eq. (35a) and the system-scale prediction Eq. (38a) that both naturally arise in the $\mathrm{Nu} \approx \mathrm{Pe}_{\mathrm{TW}}$ rapidly rotating scaling turbulent arguments given here and in rapidly rotating asymptotic analysis [17,55,91,99]. Directly comparing the Reynolds numbers scalings in Eqs. (35a) and (47a) is, however, incorrect since they are defined on different length scales. In contrast, it is appropriate to compare Eqs. (38a) and (35a) since they are both system-scale quantities, and we have shown, in fact, that these scalings are identical in the turbulent RRL where $\mathrm{Nu} \rightarrow \mathrm{Pe}_{\mathrm{TW}}$. 
TABLE I. Summary of applied $\Delta T$, turbulent scaling estimates for characteristic convective scales and transports in the slowly rotating limit (SRL) and the rapidly rotating limit (RRL). The free fall velocity is defined here as $U_{f f} \sim \sqrt{g \alpha \Delta T H}$ and $\operatorname{Ro}_{\ell} \sim \operatorname{Ro}_{C}=\sqrt{\operatorname{RaEk}^{2} / \operatorname{Pr}_{1}}$ both SRL and RRL. The nonrotating (NRL) scalings are identical to SRL in our treatment, excepting that $\operatorname{Ro}_{C}=\infty$ in the nonrotating regime.

\begin{tabular}{|c|c|c|c|c|c|c|c|c|c|c|}
\hline $\begin{array}{l}\text { Regime } \\
\text { ( } \Delta T \text { based) }\end{array}$ & $\begin{aligned} & \operatorname{Ro}_{\ell} \\
\approx & \operatorname{Ro}_{C}\end{aligned}$ & $\ell$ & $\vartheta$ & $U$ & $\operatorname{Re}_{\ell}$ & $\mathrm{Pe}_{\ell}$ & $\mathrm{Nu}$ & $\operatorname{Re}_{H}$ & $\mathrm{Pe}_{H}$ & $\mathrm{Ro}_{H}$ \\
\hline SRL & $\gg 1$ & $H$ & $\Delta T$ & $U_{f f}$ & $\operatorname{Re}_{f f}$ & $\mathrm{Pe}_{f f}$ & $\mathrm{Pe}_{f f}$ & $\operatorname{Re}_{f f}$ & $\mathrm{Pe}_{f f}$ & $\mathrm{Ro}_{C}$ \\
\hline RRL & $\ll 1$ & $\operatorname{Ro}_{C} H$ & $\operatorname{Ro}_{C} \Delta T$ & $\operatorname{Ro}_{C} U_{f f}$ & $\operatorname{Ro}_{C}{ }^{2} \operatorname{Re}_{f f}$ & $\operatorname{Ro}_{C}^{2} \mathrm{Pe}_{f f}$ & $\operatorname{Ro}_{C}^{2} \mathrm{Pe}_{f f}$ & $\operatorname{Ro}_{C} \operatorname{Re}_{f f}$ & $\operatorname{Ro}_{C} \operatorname{Re}_{f f}$ & $\operatorname{Ro}_{C}{ }^{2}$ \\
\hline
\end{tabular}

The respective dimensional forms of the rapidly rotating length, temperature fluctuation, temperature drop, and velocity scales are

$$
\begin{aligned}
\ell & \sim \operatorname{Ro}_{C} H \sim\left(\frac{g \alpha Q H^{3}}{8 \rho c_{p} \Omega^{3}}\right)^{1 / 5} \\
\vartheta & \sim \frac{Q}{\rho c_{P} U_{\mathrm{TW}}} \sim\left(\frac{2 \Omega}{g^{2} \alpha^{2} H}\right)^{1 / 5}\left(\frac{Q}{\rho c_{p}}\right)^{3 / 5}, \\
\Delta T & \sim \frac{\vartheta H}{\ell} \sim \frac{(2 \Omega)^{4 / 5} H^{1 / 5}}{(g \alpha)^{3 / 5}}\left(\frac{Q}{\rho c_{p}}\right)^{2 / 5}, \\
U & \sim U_{\mathrm{TW}} \sim \sqrt{g \alpha \vartheta \ell} \sim \frac{g \alpha \vartheta}{2 \Omega} \frac{H}{\ell} \\
& \sim\left(\frac{g \alpha Q}{\rho c_{p}}\right)^{2 / 5}\left(\frac{H}{2 \Omega}\right)^{1 / 5} .
\end{aligned}
$$

In this section, we have transformed the scaling results produced in the $\Delta T$-based framework to the $Q$-based framework via the definition of the flux Rayleigh number $\mathrm{Ra}_{F}=\mathrm{RaNu}$. In the flux-based scalings, we find a lack of equivalence between the SRL and RRL local Rossby numbers. Nevertheless, exploration of the flux-based framework has shown that the classical, flux-based CIA scalings produced in many prior works are formally synonymous with the temperature-based scalings developed herein [cf. Eqs. (39) and (49)].

\section{DISCUSSION}

The convective scaling relationships presented here are generated via exactly parallel constructions, first made within the nonrotating and SRLs and then secondarily made within the RRL. Starting from the generic nondimensional transport parameters, $\mathrm{Re}=U \ell / \nu, \mathrm{Pe}=U \ell / \kappa$, and $\mathrm{Nu}=$ $U \vartheta /(\kappa \Delta T / H)$, we select the dynamically relevant estimates for $\ell, \vartheta$, and $U$ that characterize a given convection system. Two configurations of thermal driving are considered: the fixed-temperature regime (Table I), popular for its ease of application and interpretation in modeling studies, and the fixed heat flux regime (Table II), popular for its ease of application in geophysical and astrophysical settings.

The fixed-temperature configuration is particularly elegant, and we will focus on the fixed temperature scalings in this discussion. First, our analyses show that the local Rossby number is equivalent to the convective Rossby number,

$$
\mathrm{Ro}_{\ell} \simeq \operatorname{Ro}_{C},
$$

in both the slowly and the rapidly rotating frameworks, where $\operatorname{Ro}_{\ell} \equiv U /(2 \Omega \ell)$ is estimated using the characteristic convective length $\ell$, the velocity scale $U$ for each limit, and $\operatorname{Ro}_{C} \equiv \sqrt{\operatorname{RaEk}^{2} \operatorname{Pr}^{-1}}$.

Second, by taking the ratios of the rapidly rotating and slowly rotating characteristic scales, we find that they are all related via powers of $\operatorname{Ro}_{C}{ }^{1}$,

$$
\frac{\ell}{H} \sim \frac{\vartheta}{\Delta T} \sim \frac{U_{\mathrm{TW}}}{U_{f f}} \sim \operatorname{Ro}_{C} .
$$

Third, we have shown that the RRL thermal wind transports and the SRL free-fall transports differ from one another via powers of $\operatorname{Ro}_{C}{ }^{2}$,

$$
\frac{\mathrm{Re}_{\mathrm{TW}}}{\mathrm{Re}_{f f}} \sim \frac{\mathrm{Pe}_{\mathrm{TW}}}{\mathrm{Pe}_{f f}} \sim \mathrm{Ro}_{C}{ }^{2} .
$$

Further, our generic scalings predict that the system-scale Rossby number, $\mathrm{Ro}_{H}$, scales as $\mathrm{Ro}_{C}$ in the slowly rotating regime and as $\mathrm{Ro}_{C}{ }^{2}$ in the rapidly rotating regime. Thus, the convective Rossby number is shown to explain the localscale convection dynamics, $\mathrm{Ro}_{\ell} \approx \mathrm{Ro}_{C}$, and is essential for relating the slowly rotating convection behaviors to those of the rapidly rotating regime. $\mathrm{Ro}_{C}$, and synonymously $\mathrm{Ro}_{\ell}$, arise ubiquitously in describing rotating convective flows. Furthermore, the theoretical framework we have developed

\begin{tabular}{|c|c|c|c|c|c|c|c|c|c|c|}
\hline $\begin{array}{l}\text { Regime } \\
(Q \text {-based) }\end{array}$ & $\begin{aligned} & R o_{\ell} \\
\approx & \operatorname{Ro}_{C}\end{aligned}$ & $\ell$ & $\vartheta$ & $U$ & $\operatorname{Re}_{\ell}$ & $\mathrm{Pe}_{\ell}$ & $\mathrm{Nu}$ & $\mathrm{Re}_{H}$ & $\mathrm{Pe}_{H}$ & $\mathrm{Ro}_{H}$ \\
\hline SRL & $\gg 1$ & $H$ & $\Delta T$ & $U_{f f}$ & $\operatorname{Re}_{f f}$ & $\mathrm{Pe}_{f f}$ & $\mathrm{Pe}_{f f}$ & $\operatorname{Re}_{f f}$ & $\mathrm{Pe}_{f f}$ & $\operatorname{Ro}_{C}$ \\
\hline RRL & $\ll 1$ & $\operatorname{Ro}_{C} H$ & $\operatorname{Ro}_{C} \Delta T$ & $\operatorname{Ro}_{C}{ }^{1 / 3} U_{f f}$ & $\operatorname{Ro}_{C}{ }^{4 / 3} \operatorname{Re}_{f f}$ & $\operatorname{Ro}_{C}{ }^{4 / 3} \mathrm{Pe}_{f f}$ & $\operatorname{Ro}_{C}{ }^{4 / 3} \mathrm{Pe}_{f f}$ & $\operatorname{Ro}_{C}^{1 / 3} \operatorname{Re}_{f f}$ & $\operatorname{Ro}_{C}^{1 / 3} \operatorname{Re}_{f f}$ & $\operatorname{Ro}_{C}^{2}$ \\
\hline
\end{tabular}
here provides a remarkably straightforward set of experimentally testable interconnections between the slowly rotating and rapidly rotating convective regimes. As summarized in

TABLE II. Summary of applied $Q$, turbulent scaling estimates for characteristic convective scales and transports in the slowly rotating limit (SRL) and the rapidly rotating limit (RRL). The free fall velocity is defined here as $U_{f f} \sim\left(g \alpha Q H / \rho c_{P}\right)^{1 / 3}=(\mathcal{B} H)^{1 / 3}$. Note in the flux based framework that $\operatorname{Ro}_{\ell} \sim \operatorname{Ro}_{C} \sim \mathrm{Ra}_{F}^{* 1 / 3}$ in the SRL, whereas $\operatorname{Ro}_{\ell} \sim \operatorname{Ro}_{C} \sim \mathrm{Ra}_{F}^{* 1 / 5}$ in the RRL. Thus, in the rapidly rotating regime $\mathrm{Ro}_{H} \sim \operatorname{Ro}_{C}{ }^{2} \sim \mathrm{Ra}_{F}^{* 2 / 5}$, consistent with $\Delta T \sim Q^{2 / 5}$ in Eq. (50c). 
Tables I and II, these scalings all depend rather simply on the relevant free-fall transport parameter and on $\mathrm{Ro}_{\ell} \approx \mathrm{Ro}_{C}$.

We have shown that when $\mathrm{Ro}_{\ell}$ is defined using the appropriate slowly rotating characteristic scales is equivalent to the convective Rossby number $\operatorname{Ro}_{C}$ :

$$
\mathrm{Ro}_{\ell}=\frac{U_{f f}}{2 \Omega H}=\frac{\tau_{\Omega}}{\tau_{f f}}=\sqrt{\frac{\mathrm{RaEk}^{2}}{\operatorname{Pr}}} \equiv \operatorname{Ro}_{C}(\mathrm{SRL}) .
$$

Following from this, $\mathrm{Ro}_{C}$ is often interpreted as the ratio between freely falling convective inertia and the system's rotational inertia $[20,39,40,45]$. This interpretation is accurate in the slowly rotating regime $[44,46,66]$. In contrast, this $U_{f f^{-}}$ based interpretation is not accurate in rapidly rotating cases, where the length and velocities scales are far smaller than in the slowly rotating regime (Table I).

Surprisingly, though, we have shown that the $\mathrm{Ro}_{\ell}$ also scales equivalently to $\operatorname{Ro}_{C}$ in the RRL:

$$
\mathrm{Ro}_{\ell}=\frac{U_{\mathrm{TW}}}{2 \Omega \ell}=\frac{\tau_{\Omega}}{\tau_{\mathrm{TW}}}=\sqrt{\frac{\mathrm{RaEk}^{2}}{\operatorname{Pr}}} \equiv \mathrm{Ro}_{C}(\mathrm{RRL}) .
$$

This equivalence holds since the free-fall timescale in the slowly rotating regime scales similarly to the thermal wind timescale in the rapidly rotating regime:

$$
\tau_{f f}=\frac{H}{U_{f f}} \sim \frac{\ell}{U_{\mathrm{TW}}}=\tau_{\mathrm{TW}} .
$$

The Rossby number based on the dominant dynamical scale is equivalent to the convective Rossby number in both end member rotational regimes, $R o_{\ell} \simeq \operatorname{Ro}_{C}$. This makes clear that the convective Rossby number is, in fact, an appropriate descriptor of rapidly rotating convection dynamics, but it should always be cast as $\operatorname{Ro}_{C}=U_{\mathrm{TW}} /(2 \Omega \ell)$ in the RRL. Further, since $\mathrm{Ro}_{\ell} \simeq \mathrm{Ro}_{C}$ in both regimes, $\mathrm{Ro}_{C}$ can be further interpreted as the descriptor of the local scale rotating convection dynamics, irrespective of its value. We conclude then that the convective Rossby number is rather aptly named.

The fixed heat flux configuration can be deduced from the fixed-temperature configuration through the relation $\mathrm{Ra}_{F}=$ $\mathrm{RaNu}$. We again find that $\mathrm{Ro}_{\ell} \simeq \mathrm{Ro}_{C}$ in both the slow rotating and RRLs. However, they no longer have a common definition: $\operatorname{Ro}_{C} \sim \operatorname{Ra}_{F}^{* 1 / 3}$ in the SRL regime and $\operatorname{Ro}_{C} \sim \operatorname{Ra}_{F}^{* 1 / 5}$ in the RRL regime. The relationships between the various flux-based scalings are given in Table II.

Irrespective of the configuration, a clear interpretation of $\operatorname{Ro}_{C}$ arises from our scaling analyses. The two characteristic velocities in rotating convection are $U_{f f}$ and $U_{\mathrm{TW}}$. In slowly rotating convection, $U \sim U_{f f} \ll U_{\mathrm{TW}}$, since all the fluid's buoyant potential energy is converted to kinetic energy well before it reaches $U_{\mathrm{TW}}$. (Alternatively stated, $U_{\mathrm{TW}}$ becomes singularly large as $\Omega$ becomes small.) In rapidly rotating convection, $U \sim U_{\mathrm{TW}} \ll U_{f f}$ since the vortex stretching term in Eq. (23) greatly limits the distance through which a rotating parcel of buoyant fluid can actually freely fall [83]. The selection between $U_{f f}$ and $U_{\mathrm{TW}}$ is based on the more restrictive value between the two:

$$
U \simeq \min \left(U_{f f}, U_{\mathrm{TW}}\right) .
$$

Since $\operatorname{Ro}_{C}=U_{\mathrm{TW}} / U_{f f}$, it can be validly interpreted as the essential control parameter that picks between the two characteristic velocitites:

$$
\begin{aligned}
& \operatorname{Ro}_{C} \gg 1 \Rightarrow \min \left(U_{f f}, U_{\mathrm{TW}}\right)=U_{f f}, \\
& \operatorname{Ro}_{C} \sim 1 \Rightarrow \min \left(U_{f f}, U_{\mathrm{TW}}\right)=U, \\
& \operatorname{Ro}_{C} \ll 1 \Rightarrow \min \left(U_{f f}, U_{\mathrm{TW}}\right)=U_{\mathrm{TW}} .
\end{aligned}
$$

The relative ordering of the characteristic time scales is also, therefore, set by $\mathrm{Ro}_{C}$ :

$$
\begin{aligned}
& \operatorname{Ro}_{C} \gg 1 \Rightarrow \tau_{\Omega} \gg\left(\tau_{f f} \sim \tau_{U}^{H}\right), \\
& \operatorname{Ro}_{C} \sim 1 \Rightarrow \tau_{\Omega} \sim \tau_{f f} \sim \tau_{U}^{\ell} \sim \tau_{U}^{H}, \\
& \operatorname{Ro}_{C} \ll 1 \Rightarrow \tau_{\Omega} \ll\left(\tau_{f f} \sim \tau_{U}^{\ell}\right) \ll \tau_{U}^{H} .
\end{aligned}
$$

The intermediate $\operatorname{Ro}_{C} \sim 1$ regime has not been considered here. There is, however, a great deal of laboratory data $[22,41,42,80,100-102]$ and numerical simulation data $[25,39,40,45-47,51,84,103-106]$ in the $\operatorname{Ro}_{C}=\mathcal{O}(1)$ regime. Thus, its scaling behaviors are of broad interest and should be considered in future studies.

An array of new convection and rotating convection devices have been recently built at research centers worldwide $[15,63,107]$. These next-generation laboratory devices and associated state-of-the-art numerical simulations, will allow investigations into the efficacy and applicability ranges of the turbulent scaling predictions presented here (Tables I and II). Our goal will then be to test, possibly validate, and disambiguate between these differing scaling laws given high fidelity measurements, and thereby deduce accurate, robust relations for nonrotating, slowly rotating, and rapidly rotating convective heat and momentum transport, as is necessary to explain and interpret industrial, astrophysical and geophysical convection phenomena.

\section{ACKNOWLEDGMENTS}

This work arose from discussions at the workshop "Rotating Convection: From the Lab to the Stars" held at the Lorentz Center in May 2018. We gratefully acknowledge the financial support of the NSF Geophysics Program (EAR Awards No. 1620649 and 1853196), NSF Applied Mathematics Program (DMS Award No. 2009319), NSF Astronomical Sciences (AST Award No. 1821988), NASA (Award No. 80NSSC17K0008) and the German Research Foundation (DFG Award No. HO 5890/1-1). Further thanks are given to Jewel Abbate (UCLA) and Thomas Gastine (IPGP) for supplying the images used in Figs. 2 and 3, and for referee comments that greatly improved this paper.
[1] G. Ahlers, S. Grossmann, and D. Lohse, Heat transfer and large scale dynamics in turbulent Rayleigh-Bénard convection, Rev. Mod. Phys. 81, 503 (2009).
[2] E. M. King and B. A. Buffett, Flow speeds and length scales in geodynamo models: The role of viscosity, Earth Planet. Sci. Lett. 371-372, 156 (2013). 
[3] I. Grooms and J. Whitehead, Bounds on heat transport in rapidly rotating Rayleigh-Bénard convection, Nonlinearity 28, 29 (2014).

[4] M. Plumley and K. Julien, Scaling laws in Rayleigh-Bénard convection, Earth and Space Sci. 6(9), 1580 (2019).

[5] D. Stevenson, Turbulent thermal convection in the presence of rotation and a magnetic field: A heuristic theory, Geophys. Astrophys. Fluid Dyn. 12, 139 (1979).

[6] G. Glatzmaier, Geodynamo simulations-how realistic are they? Annu. Rev. Earth Planet Sci. 30, 237 (2002).

[7] P. Diamond, C. McDevitt, O. Gürcan, T. Hahm, W. Wang, E. Yoon, I. Holod, Z. Lin, V. Naulin, and R. Singh, Physics of non-diffusive turbulent transport of momentum and the origins of spontaneous rotation in tokamaks, Nucl. Fusion 49, 045002 (2009).

[8] P. H. Roberts and E. M. King, On the genesis of the Earth's magnetism, Rev. Prog. Phys. 76(9), 096801 (2013).

[9] Ö. Gürcan and P. Diamond, Zonal flows and pattern formation, J. Phys. A: Math. Theor. 48, 293001 (2015).

[10] N. Featherstone and B. Hindman, The spectral amplitude of stellar convection and its scaling in the high-Rayleigh-number regime, Astrophys. J. 818, 32 (2016).

[11] H. Cao, R. Yadav, and J. Aurnou, Geomagnetic polar minima do not arise from steady meridional circulation, Proc. Natl. Acad. Sci. USA 115, 11186 (2018).

[12] T. Wood, P. Garaud, and S. Stellmach, A new model for mixing by double-diffusive convection (semi-convection). II. The transport of heat and composition through layers, Astrophys. J. 768, 157 (2013).

[13] S. Horn and J. Aurnou, Regimes of Coriolis-Centrifugal Convection, Phys. Rev. Lett. 120, 204502 (2018).

[14] K. Julien and E. Knobloch, Strongly nonlinear convection cells in a rapidly rotating fluid layer: The tilted $f$-plane, J. Fluid Mech. 360, 141 (1998).

[15] J. Cheng, J. Aurnou, K. Julien, and R. Kunnen, A heuristic framework for next-generation models of geostrophic convective turbulence, Geophys. Astrophys. Fluid Dyn. 112, 277 (2018).

[16] P. Marti, M. Calkins, and K. Julien, A computationally efficient spectral method for modeling core dynamics, Geochem. Geophys. Geosys. 17, 3031 (2016).

[17] M. Sprague, K. Julien, E. Knobloch, and J. Werne, Numerical simulation of an asymptotically reduced system for rotationally constrained convection, J. Fluid Mech. 551, 141 (2006).

[18] E. King and J. Aurnou, Magnetostrophic balance as the optimal state for turbulent magnetoconvection, Proc. Natl. Acad. Sci. USA 112, 990 (2015).

[19] S. Horn and J. Aurnou, Rotating convection with centrifugal buoyancy: Numerical predictions for laboratory experiments, Phys. Rev. Fluids 4, 073501 (2019).

[20] S. Horn and O. Shishkina, Rotating non-Oberbeck-Boussinesq Rayleigh-Bénard convection in water, Phys. Fluids 26, 055111 (2014).

[21] M. A. Calkins, K. Hale, K. Julien, D. Nieves, D. Driggs, and P. Marti, The asymptotic equivalence of fixed heat flux and fixed temperature thermal boundary conditions for rapidly rotating convection, J. Fluid Mech. 784, R2 (2015).

[22] J. S. Cheng, S. Stellmach, A. Ribeiro, A. Grannan, E. M. King, and J. M. Aurnou, Laboratory-numerical models of rapidly rotating convection in planetary cores, Geophys. J. Int. 201, 1 (2015).

[23] N. Gillet and C. Jones, The quasi-geostrophic model for rapidly rotating spherical convection outside the tangent cylinder, J. Fluid Mech. 554, 343 (2006).

[24] U. Christensen, Zonal flow driven by strongly supercritical convection in rotating spherical shells, J. Fluid Mech. 470, 115 (2002).

[25] U. Christensen and J. Aubert, Scaling properties of convection-driven dynamos in rotating spherical shells and application to planetary magnetic fields, Geophys. J. Int. 166, 97 (2006)

[26] T. Maxworthy and S. Narimousa, Unsteady, turbulent convection into a homogeneous, rotating fluid, with oceanographic applications, J. Phys. Oceanogr. 24, 865 (1994).

[27] S. Chandrasekhar, Hydrodynamic and Hydromagnetic Stability (Oxford University Press, Oxford, 1961).

[28] K. Julien and E. Knobloch, Fully nonlinear three-dimensional convection in a rapidly rotating layer, Phys. Fluids 11, 1469 (1999).

[29] K. Zhang and X. Liao, Theory and Modeling of Rotating Fluids: Convection, Inertial Waves and Precession (Cambridge University Press, Cambridge, 2017).

[30] C. Guervilly and P. Cardin, Subcritical convection of liquid metals in a rotating sphere using a quasi-geostrophic model, J. Fluid Mech. 808, 61 (2016).

[31] E. Kaplan, N. Schaeffer, J. Vidal, and P. Cardin, Subcritical Thermal Convection of Liquid Metals in a Rapidly Rotating Sphere, Phys. Rev. Lett. 119, 094501 (2017).

[32] E. Sparrow, R. Goldstein, and V. Jonsson, Thermal instability in a horizontal fluid layer: Effect of boundary conditions and non-linear temperature profile, J. Fluid Mech. 18, 513 (1964).

[33] S. Horn and P. Schmid, Prograde, retrograde, and oscillatory modes in rotating Rayleigh-Bénard convection, J. Fluid Mech. 831, 182 (2017).

[34] J. Aurnou, V. Bertin, A. Grannan, S. Horn, and T. Vogt, Rotating thermal convection in liquid gallium: Multi-modal flow, absent steady columns, J. Fluid Mech. 846, 846 (2018).

[35] S. Chandrasekhar and D. Elbert, The instability of a layer of fluid heated below and subject to Coriolis forces. II, Proc. R. Soc. London A 231, 198 (1955).

[36] K. Julien and E. Knobloch, Fully nonlinear oscillatory convection in a rotating layer, Phys. Fluids 9, 1906 (1997).

[37] C. Jones, A. Soward, and A. Mussa, The onset of thermal convection in a rapidly rotating sphere, J. Fluid Mech. 405, 157 (2000).

[38] J. Dawes, Rapidly rotating thermal convection at low Prandtl number, J. Fluid Mech. 428, 61 (2001).

[39] P. Gilman, Nonlinear dynamics of Boussinesq convection in a deep rotating spherical shell-i, Geophys. Astrophys. Fluid Dyn. 8, 93 (1977).

[40] K. Julien, S. Legg, J. McWilliams, and J. Werne, Rapidly rotating turbulent Rayleigh-Bénard convection, J. Fluid Mech. 322, 243 (1996).

[41] R. Kunnen, H. Clercx, and B. Geurts, Breakdown of largescale circulation in turbulent rotating convection, Europhys. Lett. 84, 24001 (2008). 
[42] S. Weiss and G. Ahlers, Heat transport by turbulent rotating Rayleigh-Bénard convection and its dependence on the aspect ratio, J. Fluid Mech. 684, 407 (2011).

[43] R. J. A. M. Stevens, E. P. van der Poel, S. Grossmann, and D. Lohse, The unifying theory of scaling in thermal convection: the updated prefactors, J. Fluid Mech. 730, 295 (2013).

[44] J. Zhong, H. Li, and X. Wang, Enhanced azimuthal rotation of the large-scale flow through stochastic cessations in turbulent rotating convection with large Rossby numbers, Phys. Rev. Fluids 2, 044602 (2017).

[45] J. Aurnou, M. Heimpel, and J. Wicht, The effects of vigorous mixing in a convective model of zonal flow on the ice giants, Icarus 190, 110 (2007).

[46] T. Gastine, J. Wicht, and J. Aurnou, Zonal flow regimes in rotating anelastic spherical shells: An application to giant planets, Icarus 225, 156 (2013).

[47] T. Gastine, R. K. Yadav, J. Morin, A. Reiners, and J. Wicht, From solar-like to antisolar differential rotation in cool stars, Mon. Not. R. Astron. Soc. 438, L76 (2013).

[48] E. King and J. Aurnou, Turbulent convection in liquid metal with and without rotation, Proc. Natl. Acad. Sci. USA 110, 6688 (2013).

[49] K. Soderlund, B. Schmidt, J. Wicht, and D. Blankenship, Ocean-driven heating of Europa's icy shell at low latitudes, Nat. Geosci. 7, 16 (2014).

[50] S. Horn and O. Shishkina, Toroidal and poloidal energy in rotating Rayleigh-Bénard convection, J. Fluid Mech. 762, 232 (2015).

[51] J. Mabuchi, Y. Masada, and A. Kageyama, Differential rotation in magnetized and non-magnetized stars, Astrophys. J. 806, 10 (2015).

[52] J. Cheng, M. Madonia, A. Aguirre Guzmán, and R. Kunnen, Laboratory Exploration of Heat Transfer Regimes in Rapidly Rotating Turbulent Convection, arXiv:1911.04537v4 (2020).

[53] J. Zhong, R. Stevens, H. Clercx, R. Verzicco, D. Lohse, and G. Ahlers, Prandtl-, Rayleigh-, and Rossby-Number Dependence of Heat Transport in Turbulent Rotating Rayleigh-Bénard Convection, Phys. Rev. Lett. 102, 044502 (2009).

[54] J. Cheng and J. Aurnou, Tests of diffusion-free scaling behaviors in numerical dynamo datasets, Earth Planet. Sci. Lett. 436, 121 (2016).

[55] M. Calkins, Quasi-geostrophic dynamo theory, Phys. Earth Planet. Inter. 276, 182 (2018).

[56] R. Ecke and J. Niemela, Heat Transport in the Geostrophic Regime of Rotating Rayleigh-Bénard Convection, Phys. Rev. Lett. 113, 114301 (2014).

[57] S. Grossmann and D. Lohse, Scaling in thermal convection: A unifying theory, J. Fluid Mech. 407, 27 (2000).

[58] F. Chillà and J. Schumacher, New perspectives in turbulent Rayleigh-Bénard convection, Eur. Phys. J. E 35, 1 (2012).

[59] M. Verma, Physics of Buoyant Flows: From Instabilities to Turbulence (World Scientific, London, 2018).

[60] J. Glazier, T. Segawa, A. Naert, and M. Sano, Evidence against 'ultrahard' thermal turbulence at very high Rayleigh numbers, Nature 398, 307 (1999).

[61] D. Lohse and F. Toschi, Ultimate State of Thermal Convection, Phys. Rev. Lett. 90, 034502 (2003).

[62] X. He, D. Funfschilling, H. Nobach, E. Bodenschatz, and G. Ahlers, Transition to the Ultimate State of Turbulent RayleighBénard Convection, Phys. Rev. Lett. 108, 024502 (2012).
[63] S. Lepot, S. Aumaître, and B. Gallet, Radiative heating achieves the ultimate regime of thermal convection, Proc. Natl. Acad. Sci. USA 115, 8937 (2018).

[64] C. Doering, S. Toppaladoddi, and J. Wettlaufer, Abscence of Evidence for the Ultimate Regime in Two-Dimensional Rayleigh-Bénard Convection, Phys. Rev. Lett. 123, 259401 (2019).

[65] X. Zhu, V. Mathai, R. Stevens, R. Verzicco, and D. Lohse, Reply to Abscence of Evidence for the Ultimate Regime in Two-Dimensional Rayleigh-Bénard Convection, Phys. Rev. Lett. 123, 259402 (2019).

[66] E. Brown and G. Ahlers, Effect of the Earth's Coriolis force on the large-scale circulation of turbulent Rayleigh-Bénard convection, Phys. Fluids 18, 125108 (2006).

[67] T. Gastine, J. Wicht, and J. Aurnou, Turbulent RayleighBénard convection in spherical shells, J. Fluid Mech. 778, 721 (2015).

[68] J. Von Hardenberg, A. Parodi, G. Passoni, A. Provenzale, and E. Spiegel, Large-scale patterns in Rayleigh-Bénard convection, Phys. Lett. A 372, 2223 (2008).

[69] P. Sakievich, Y. Peet, and R. Adrian, Large-scale thermal motions of turbulent Rayleigh-Bénard convection in a wide aspect-ratio cylindrical domain, Int. J. Heat Fluid Flow 61, 183 (2016).

[70] A. Pandey, J. Scheel, and J. Schumacher, Turbulent superstructures in Rayleigh-Bénard convection, Nat. Commun. 9, 2118 (2018)

[71] T. Vogt, S. Horn, A. Grannan, and J. Aurnou, Jump rope vortex in liquid metal convection, Proc. Natl. Acad. Sci. USA 115, 12674 (2018).

[72] M. Akashi, T. Yanagisawa, Y. Tasaka, T. Vogt, Y. Murai, and S. Eckert, Transition from convection rolls to large-scale cellular structures in turbulent Rayleigh-Bénard convection in a liquid metal layer, Phys. Rev. Fluids 4, 033501 (2019).

[73] M. Rieutord and F. Rincon, The Sun's supergranulation, Living Rev. Sol. Phys. 7, 2 (2010).

[74] S. Grossmann and D. Lohse, Multiple scaling in the ultimate regime of thermal convection, Phys. Fluids 23, 045108 (2011).

[75] E. Spiegel, A generalization of the mixing-length theory of turbulent convection, Astrophys. J. 138, 216 (1963).

[76] E. A. Spiegel, Convection in stars I. Basic boussinesq convection, Annu. Rev. Astron. Astrophys. 9, 323 (1971).

[77] S. Grossmann and D. Lohse, Fluctuations in turbulent Rayleigh-Bénard convection: The role of plumes, Phys. Fluids 16, 4462 (2004).

[78] C. Doering, Turning up the heat in turbulent thermal convection, Proc. Natl. Acad. Sci. USA 117, 9671 (2020).

[79] R. Ecke, Scaling of heat transport near onset in rapidly rotating convection, Phys. Lett. A 379, 2221 (2015).

[80] H. Greenspan, The Theory of Rotating Fluids (CUP Archive, Cambridge, 1968).

[81] A. Ingersoll and D. Pollard, Motion in the interiors and atmospheres of Jupiter and Saturn: Scale analysis, anelastic equations, barotropic stability criterion, Icarus 52, 62 (1982).

[82] J. Aubert, D. Brito, H.-C. Nataf, P. Cardin, and J.-P. Masson, A systematic experimental study of rapidly rotating spherical convection in water and liquid gallium, Phys. Earth Planet. Inter. 128, 51 (2001)

[83] C. A. Jones, Planetary magnetic fields and fluid dynamos, Annu. Rev. Fluid Mech. 43, 583 (2011). 
[84] T. Gastine, J. Wicht, and J. Aubert, Scaling regimes in spherical shell rotating convection, J. Fluid Mech. 808, 690 (2016).

[85] S. Stellmach and U. Hansen, Cartesian convection driven dynamos at low Ekman number, Phys. Rev. E 70, 056312 (2004).

[86] K. Julien, A. Rubio, I. Grooms, and E. Knobloch, Statistical and physical balances in low Rossby number RayleighBénard convection, Geophys. Astrophys. Fluid Dyn. 106, 392 (2012).

[87] J. Hart and D. Ohlsen, On the thermal offset in turbulent rotating convection, Phys. Fluids 11, 2101 (1999).

[88] J. McWilliams, Fundamentals of Geophysical Fluid Dynamics (Cambridge University Press, Cambridge, 2006).

[89] K. Julien, E. Knobloch, A. Rubio, and G. Vasil, Heat Transport in Low-Rossby-Number Rayleigh-Bénard Convection, Phys. Rev. Lett. 109, 254503 (2012a).

[90] A. Barker, A. Dempsey, and Y. Lithwick, Theory and simulations of rotating convection, Astrophys. J. 791, 13 (2014).

[91] S. Maffei, K. Julien, P. Marti, and M. Calkins, Systematic investigation of the inverse cascade and ow speeds in rapidly rotating Rayleigh-Bénard convection, arXiv:2003.01669v1 (2020).

[92] M. Plumley, K. Julien, P. Marti, and S. Stellmach, The effects of Ekman pumping on quasi-geostrophic Rayleigh-Bénard convection, J. Fluid Mech. 803, 51 (2016).

[93] M. Plumley, K. Julien, P. Marti, and S. Stellmach, Sensitivity of rapidly rotating Rayleigh-Bénard convection to Ekman pumping, Phys. Rev. Fluids 2, 094801 (2017).

[94] C. Guervilly, P. Cardin, and N. Schaeffer, Turbulent convective length scale in planetary cores, Nature 570, 368 (2019).

[95] J. W. Deardorff, Convective velocity and temperature scales for the unstable planetary boundary layer and for Rayleigh convection, J. Atmos. Sci. 27, 1211 (1970).

[96] U. Christensen, Dynamo scaling laws and applications to the planets, Space Sci. Rev. 152, 565 (2010).
[97] V. Bouillaut, S. Lepot, S. Aumaître, and B. Gallet, Transition to the ultimate regime in a radiatively driven convection experiment, J. Fluid Mech. 861, R5 (2019).

[98] K. Julien, J. Aurnou, M. Calkins, E. Knobloch, P. Marti, S. Stellmach, and G. Vasil, A nonlinear model for rotationally constrained convection with Ekman pumping, J. Fluid Mech. 798, 50 (2016).

[99] M. Calkins, K. Julien, S. Tobias, and J. Aurnou, A multiscale dynamo model driven by quasi-geostrophic convection, J. Fluid Mech. 780, 143 (2015).

[100] H. Rossby, A study of Bénard convection with and without rotation, J. Fluid Mech. 36, 309 (1969).

[101] Y. Liu and R. Ecke, Heat Transport Scaling in Turbulent Rayleigh-Bénard Convection: Effects of Rotation and Prandtl Number, Phys. Rev. Lett. 79, 2257 (1997).

[102] E. King, S. Stellmach, J. Noir, U. Hansen, and J. Aurnou, Boundary layer control of rotating convection systems, Nature 457, 301 (2009).

[103] K. Soderlund, E. King, and J. Aurnou, The influence of magnetic fields in planetary dynamo models, Earth Planet. Sci. Lett. 333, 9 (2012).

[104] N. Featherstone and M. Miesch, Meridional circulation in solar and stellar convection zones, Astrophys. J. 804, 67 (2015).

[105] E. Anders, C. Manduca, B. Brown, J. Oishi, and G. Vasil, Predicting the Rossby number in convective experiments, Astrophys. J. 872, 138 (2019).

[106] R. Long, J. Mound, C. Davies, and S. Tobias, Scaling behavior in spherical shell rotating convection with fixed-flux thermal boundary conditions, J. Fluid Mech. 889, A7 (2020).

[107] T. Zürner, F. Schindler, T. Vogt, S. Eckert, and J. Schumacher, Combined measurement of velocity and temperature in liquid metal convection, J. Fluid Mech. 876, 1108 (2019).

Correction: The NASA Award number given in the Acknowledgment section contained an error and has been fixed. 